\section{SAT0173 1 A SYSTEMATIC LITERATURE REVIEW INFORMING THE 2019 UPDATE OF THE JOINT EUROPEAN LEAGUE AGAINST RHEUMATISM AND EUROPEAN RENAL ASSOCIATION-EUROPEAN DIALYSIS AND TRANSPLANT ASSOCIATION (EULAR/ERA-EDTA) RECOMMENDATIONS FOR THE MANAGEMENT OF LUPUS NEPHRITIS}

A. Fanouriakis ${ }^{1}$, M. Kostopoulou' ${ }^{2}$, K. Cheema ${ }^{3}$, G. Bertsias ${ }^{4}$, D. Jayne ${ }^{3}$, D. Boumpas ${ }^{5}$. 'University of Athens, Athens, Greece; " "G. Gennimatas" General Hospital, Athens, Greece; ${ }^{3}$ University of Cambridge, Cambridge, United Kingdom; ${ }^{4}$ University of Crete Medical School, Heraklion, Greece; ${ }^{5}$ University of Athens Medical School, Athens, Greece

Background: Lupus nephritis (LN) affects $~ 40 \%$ of patients with systemic lupus erythematosus (SLE) and is associated with significant morbidity. New data has emerged since the publication of the EULAR/ERA-EDTA recommendations for the management of $L N$, involving a multidisciplinary panel of experts.

Objectives: To analyze the current evidence, in order to inform the 2019 update of the EULAR/ERA-EDTA recommendations for the management of LN.

Methods: According to the EULAR standardised operating procedures, a Medline systematic literature review (SLR) was performed, from January 2012 unti 31 December 2018. The final level of evidence (LoE) and grading of recommendations considered the total body of evidence, including the LoE of the 2012 recommendations.

Results: We identified 542 relevant articles. High-quality evidence supports the use of immunosuppressive treatment for class III and IV LN (LoE 1a) there is moderate quality evidence for immunosuppression in pure class $V L N$, with nephrotic-range proteinuria (LoE 2b). Treatment should aim for a $25 \%$ reduction in proteinuria at 3 months, $50 \%$ at 6 months and complete renal response (< $500-700 \mathrm{mg} /$ day) at 12 months (LoE 2a-2b). Strong evidence supports the use of mycophenolate mofetil/mycophenolic acid (MMF/MPA) or low-dose intravenous cyclophosphamide (CY) for the initial treatment of class III/IV LN (LoE 1a, Table); Combination of tacrolimus with MMF/MPA and high-dose CY are alternatives in specific circumstances (LoE 1a, Table). There is little evidence to guide optimal duration of immunosuppression in LN (LoE 3). In end-stage kidney disease due to $L N$, all methods of kidney replacement treatment have been used, but transplantation is accompanied by the most favourable outcomes (LoE 2b).

Conclusion: There is high-quality evidence to guide the initial and subsequent phases of class III/IV LN treatment. There is low quality evidence to guide treatment of class $\mathrm{V}$, monitoring and optimal duration of immunosuppression.
Disclosure of Interests: : Antonis Fanouriakis Paid instructor for: Paid instructor for Enorasis, Amgen, Speakers bureau: Paid speaker for Roche, Genesis Pharma, Mylan, Myrto Kostopoulou: None declared, Kim Cheema: None declared, George Bertsias Grant/research support from: GSK, Consultant of: Novartis, David Jayne Grant/research support from: ChemoCentryx, GSK, Roche/Genentech, Sanofi-Genzyme, Consultant of: Astra-Zeneca, ChemoCentryx, GSK, InflaRx, Takeda, Insmed, Chugai, Boehringer-Ingelheim, Dimitrios Boumpas: None declared

DOI: 10.1136/annrheumdis-2020-eular.3936

\section{SAT0174 FLARE ASSESSMENTS IN PATIENTS WITH ACTIVE SYSTEMIC LUPUS ERYTHEMATOSUS TREATED WITH ANIFROLUMAB IN 2 PHASE 3 TRIALS}

R. Furie $^{1}$, E. F. Morand ${ }^{2}$, A. Askanase ${ }^{3}$, E. Vital ${ }^{4,5}$, R. Kalyani ${ }^{6}$, G. Abreu ${ }^{7}$, L. Pineda ${ }^{6}$, R. Tummala ${ }^{6}$. ${ }^{1}$ Zucker School of Medicine at Hofstra/Northwell, Great Neck, United States of America; ${ }^{2}$ Monash University, Melbourne, Australia; ${ }^{3}$ Columbia University College of Physicians and Surgeons, New York, United States of America; ${ }^{4}$ University of Leeds, Leeds, United Kingdom; ${ }^{5}$ NIHR Leeds Biomedical Research Centre at Leeds Teaching Hospitals NHS Trust, Leeds, United Kingdom; ${ }^{6}$ BioPharmaceuticals Medical, AstraZeneca, Gaithersburg, United States of America; ${ }^{7}$ BioPharmaceuticals Medical, AstraZeneca, Gothenburg, Sweden

Background: Anifrolumab treatment resulted in improved British Isles Lupus Assessment Group (BILAG)-based Composite Lupus Assessment (BICLA) response rates in patients with systemic lupus erythematosus (SLE) in the phase 3 TULIP-2 and TULIP-1 trials. ${ }^{1,2}$ In addition, annualized flare rates were lower among the groups treated with anifrolumab compared with placebo., ${ }^{1,2}$

Objectives: TULIP-2 and TULIP-1 data were analyzed to assess the effects of anifrolumab on the number of SLE flares and time to first flare during 52 weeks of treatment.

Methods: The randomized, double-blind, placebo-controlled TULIP-2 and TULIP-1 trials evaluated efficacy and safety of intravenous anifrolumab $300 \mathrm{mg}$ vs placebo every 4 weeks for 48 weeks, with the primary endpoints assessed at Week 52, in patients with moderate to severe SLE despite standard-of-care treatment. Flares were defined as $\geq 1$ new BILAG-2004 A or $\geq 2$ new (worsening) BILAG-2004 B domain scores compared with the prior month's visit. Time to first flare was evaluated using a Cox proportional hazards model. Annualized flare rate was analyzed using a negative binomial regression model.

Table. Randomized trials for induction therapy in LN

\begin{tabular}{|c|c|c|c|c|c|c|c|}
\hline Reference & $\mathrm{n}$ & Intervention & Control & Prednisone dosing & $\begin{array}{l}\text { End- } \\
\text { point }\end{array}$ & Results & Overall risk of bias* \\
\hline \multicolumn{8}{|l|}{ MMF vs CY } \\
\hline $\begin{array}{l}\text { Mendonca et al } \\
\text { (2017) }\end{array}$ & $40(1: 1)$ & $\mathrm{MMF}+\mathrm{Pz}$ & $\mathrm{CY}+\mathrm{Pz}$ & $0.5 \mathrm{mg} / \mathrm{kg} / \mathrm{d}$ & $\begin{array}{c}24 w \\
C R\end{array}$ & $\begin{array}{l}\text { CR: CY vs MMF } 47.82 \% \\
\text { vs } 52.94 \% p=0.861\end{array}$ & High \\
\hline & & & & & PR & $\begin{array}{c}\text { PR: CY vs MMF } 39.13 \% \\
\text { vs } 35.3 \% \text { p }=n s\end{array}$ & \\
\hline Sun et al (2015) & $82(1: 1)$ & $\mathrm{MMF}+\mathrm{CYC}+\mathrm{Pz}$ & $C Y+P z$ & $\begin{array}{c}1.0 \mathrm{mg} / \mathrm{kg} / \mathrm{d} \text { for } 4-8 \mathrm{w} \\
\text { and tapering }\end{array}$ & $6 \mathrm{~m} \mathrm{RR}$ & $\begin{array}{l}\text { RR: } \text { CY vs } \mathrm{CY}+\mathrm{MMF} \\
77.5 \% \text { vs } 88.1 \% \mathrm{p}=0.2\end{array}$ & High \\
\hline Rathi et al (2016) & $100(1: 1)$ & $\mathrm{MMF}+\mathrm{Pz}$ & $\mathrm{CY}+\mathrm{Pz}$ & $\begin{array}{l}1 \mathrm{mg} / \mathrm{kg} / \mathrm{d} \text { for } 8 \mathrm{w} \\
\text { and tapering }\end{array}$ & $\begin{array}{l}24 w \\
\text { TRR }\end{array}$ & $\begin{array}{l}\text { TRR: } \mathrm{CY} \text { vs MMF } 75.0 \% \\
\text { vs } 76.3 \% \mathrm{p}=0.91\end{array}$ & Some concerns \\
\hline Sedhain et al (2018) & $42(1: 1)$ & $\mathrm{MMF}+\mathrm{Pz}$ & $\mathrm{CY}+\mathrm{Pz}$ & $\begin{array}{c}1 \mathrm{mg} / \mathrm{kg} / \mathrm{d} \text { for } 4 \mathrm{w} \\
\text { and tapered to } \\
5-7.5 \mathrm{mg} / \mathrm{d}\end{array}$ & $6 \mathrm{~m} \mathrm{TR}$ & $\begin{array}{l}\text { TR: MMF vs CY } 28.6 \% \\
\text { vs } 19 \% p=0.57\end{array}$ & Some concerns \\
\hline Sahay et al (2018) & $\begin{array}{c}144 \\
(1: 1: 1)\end{array}$ & $\mathrm{MMF}+\mathrm{Pz}$ & $\begin{array}{l}\text { NIH regimen vs } \\
\text { Eurolupus } \\
\text { (ELNT)regimen }\end{array}$ & $\begin{array}{c}1 \mathrm{mg} / \mathrm{kg} / \mathrm{d} \text { and } \\
\text { tapered to } 10 \mathrm{mg} / \mathrm{d}\end{array}$ & $6 m R R$ & $\begin{array}{l}\text { RR: NIH:71.4\% ELNT: } \\
65 \% \text { MMF:72.9\% p=0.9 }\end{array}$ & High \\
\hline \multicolumn{8}{|l|}{ Low vs high $\mathrm{CY}$} \\
\hline Mehra et al (2018) & $77(1: 1)$ & $\begin{array}{c}\text { CY } 6 \times 500 \mathrm{mg} \text { fortnightly } \\
+\mathrm{Pz}\end{array}$ & $\begin{array}{l}\mathrm{CY} 6 \times 750 \mathrm{mg} / \mathrm{m}^{2} \\
\text { four-weekly }\end{array}$ & $\begin{array}{c}1 \mathrm{mg} / \mathrm{kg} / \mathrm{d} \text { for } 4 \mathrm{w} \\
\text { and tapered to } \\
5-7.5 \mathrm{mg} / \mathrm{d}\end{array}$ & $\begin{array}{l}52 w \\
C R \\
P R\end{array}$ & $\begin{array}{l}\text { CR: low vs high } 44 \% \text { vs } \\
65 \% p=0.08 \\
\text { CR/PR: low vs high } 50 \% \\
\text { vs } 73 \% p=0.04\end{array}$ & Some concerns \\
\hline \multicolumn{8}{|l|}{ Multitarget vs SoC } \\
\hline Liu et al (2015) & $\begin{array}{c}362 \\
(1: 1)\end{array}$ & $\mathrm{MMF}+\mathrm{TAC}+\mathrm{Pz}$ & $\mathrm{CY}+\mathrm{Pz}$ & $\begin{array}{c}0.6 \mathrm{mg} / \mathrm{kg} / \mathrm{d} \text { for } \\
4 \mathrm{w} \text { and tapered to } \\
10 \mathrm{mg} / \mathrm{d}\end{array}$ & $\begin{array}{l}24 w \\
C R \\
P R\end{array}$ & $\begin{array}{c}\text { CR: Multitarget vs CY } \\
45.9 \% \text { vs } 25.6 \% \text { p }<0.001 \\
\text { CR+PR: Multitarget vs } \\
\text { CY } 83.5 \% \text { vs } 63.0 \% \text { p } \\
0.001\end{array}$ & Some concerns \\
\hline Rovin et al (2019) & 265 & $\begin{array}{c}\text { Voclosporin (low or high } \\
\text { dose) }+ \text { MMF }+ \text { Pz }\end{array}$ & $\mathrm{MMF}+\mathrm{Pz}$ & $\begin{array}{c}20-25 \mathrm{mg} / \mathrm{d} \text { and } \\
\text { tapered to } 2.5 \mathrm{mg} / \mathrm{d} \\
\text { at } 16 \mathrm{w}\end{array}$ & $\begin{array}{l}48 w \\
\text { CRR }\end{array}$ & $\begin{array}{l}\text { CRR: low dose multitar- } \\
\text { get vs MMF OR }=3.21 \\
\qquad p<0.001 \\
\text { High dose multitarget vs } \\
\text { MMF OR=2.10 } p=0.026\end{array}$ & Low \\
\hline
\end{tabular}

